\title{
SYSTEM TO REDUCE POWER CONSUMPTION OF AIR CONDITIONERS IN VEHICLES AND DOMESTIC USES
}

\author{
Vignesh. B ${ }^{1}$, Jerry Devin. $\mathbf{J}^{2}$, Sakthi Aravindh. $\mathbf{M}^{3}$, Jones Joseph Jebaraj. $\mathrm{D}^{4}$ \\ ${ }^{1}$ Mepco Schlenk Engineering College, Tamil Nadu, India \\ ${ }^{2}$ Mepco Schlenk Engineering College, Tamil Nadu, India \\ ${ }^{3}$ Mepco Schlenk Engineering College, Tamil Nadu, India \\ ${ }^{4}$ Assistant Professor, Dept. of Mechanical Engineering, Mepco Schlenk Engineering College, Tamil Nadu, India
}

\begin{abstract}
Conventional air conditioners work based on vapor compression cycle. They draw a lot of power for their operation. This paper emphasizes a new technique which is to precool the air that enters into a conventional air conditioner using eco-friendly evaporative cooling effect. Conventional air conditioners are generally designed to recirculate $60 \%$ of indoor air and mix it with $40 \%$ of atmospheric air before passing the mixture over the evaporator coil. The process is here is to precool the atmospheric air that mixes with the recirculated air using a heat exchanger coupled with an evaporative cooler. By precooling the atmospheric air at the air intake the power consumed by the air conditioner can be reduced significantly. This will increase the overall Coefficient of Performance of the air conditioning system. Thus power to run air conditioners is saved with the effective cooling in an eco-friendly manner.
\end{abstract}

Keywords: - Evaporative cooling, Precool, Eco-Friendly, Recirculated air, Increased Coefficient of Performance. - ****

\section{INTRODUCTION}

Air conditioners are used to maintain the comfort conditions of a human being in a room. Due to global warming the average temperature of world has increased by a few degrees. This few degree rise in temperature affects a lot. To reduce the effects of global warming to comfort conditions the usage of air conditioning systems arises. The current day air conditioning systems run based on vapor compression cycle. It require a lot of power for their operation and are also responsible for a lot of carbon emissions. So there is a need fors renewable systems for air conditioning. Passive solar cooling is used in modern constructions to reduce the air conditioning requirements. Newer systems can be developed to further reduce the requirement of conventional air conditioning systems.

\section{PROBLEM IDENTIFICATION}

Conventional air conditioners operate based on vapor compression cycle. Freon's are used as refrigerants. Cooling occurs due to compression, condensation and expansion of a refrigerant in the vapor compression system. These systems operate by consuming a lot of electrical energy and this indirectly accounts to the emission of tonnes of carbon dioxide into the atmosphere. Freon's are also potent agents that increase global warming. Certain Freon's are considered to be more potent than carbon dioxide when global warming is taken into account. Also some of these substances deplete the ozone layer leading to many health complications. Thus it is seen that conventional air conditioning systems consume more power, increased global warming and also depletion of the ozone layer. This is considered as an alternative measure to reduce the requirement of conventional air conditioners.

\section{PROPOSED MODEL}

To reduce the use of conventional air conditioning systems, an eco-friendly system that works based on evaporative cooling process can be used. Evaporative cooling effect can be increased by using a venturi arrangement in a duct to reduce the pressure in the localized area. This causes more water to evaporate and causes more cooling effect. The objective of this paper is to reduce the cooling load on a conventional air conditioner by the process of evaporative cooling process. Here the cooling process is enhanced by using a venturi arrangement.

\section{PRINCIPLES USED}

\subsection{Evaporative Cooling Effect}

Evaporative cooling process works by exploiting the huge enthalpy of vaporization of water. When water is allowed to evaporate from a surface it does so by drawing heat from the surface and from the surrounding air. Similarly if water is sprayed into air it will evaporate drawing heat from the air. Thus the air cools down to lower temperatures. The temperature drop due to evaporative cooling was calculated from the psychrometric chart which is designed for atmospheric pressure. Evaporative cooling can be observed in mud pots and human's natural cooling mechanism by sweating. 


\subsection{Venturi Effect}

Venturi effect occurs when a fluid flows from a region of larger cross section to a region of smaller cross section viz. when a flowing fluid(here air) squeezes through a region of smaller cross section from a region of larger cross section, the pressure of air will drop and the velocity will increase. When there is a pressure drop, the evaporation of water also increases [1-3]. This will increase the cooling effect. By coupling both evaporative cooling effect and venturi effect, an eco-friendly air conditioning system can be created. Here the evaporative cooling effect is enhanced due to the pressure drop created by air flowing through the venturi arrangement. This means that lower temperatures can be achieved.

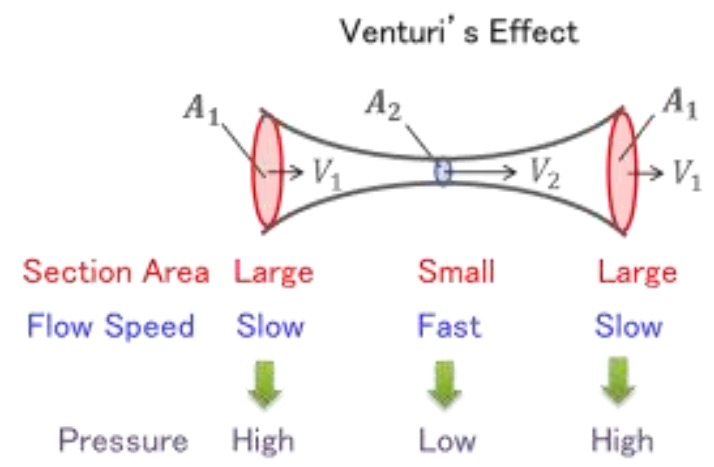

Fig -1: Venturi effect

\section{SYSTEM DESIGN}

\subsection{Duct with Venturi Arrangement}

The whole evaporative cooling process happens in a circular duct. The duct can be of any material. More preferably PVC can be used as it has good insulating properties and it is cheap and readily available. Normal air at room temperature is forced into the duct by using an axial flow fan or a centrifugal blower. A venturi arrangement is created inside the duct. This venturi arrangement can be created using metal or can be $3-D$ printed as per the requirement. This venturi arrangement reduces the pressure of air passing through the throat section.

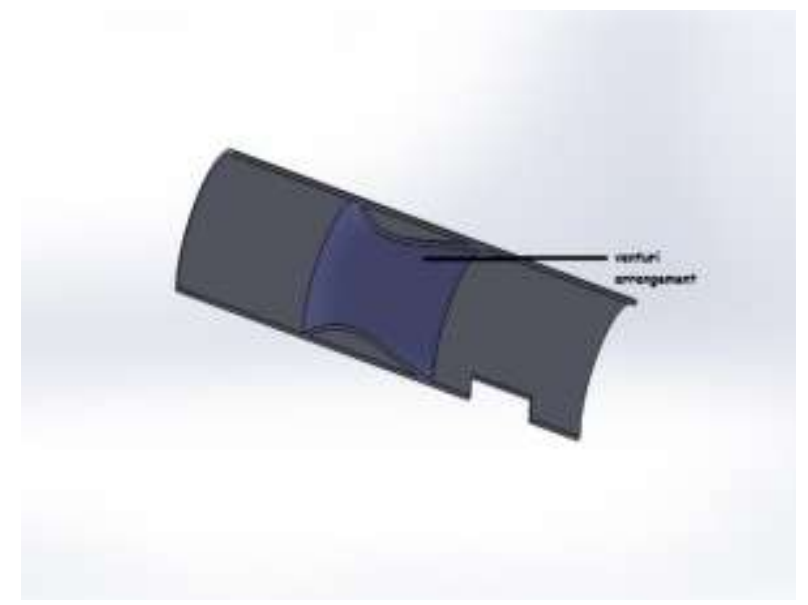

Fig -2: Venturi arrangement

\subsection{Blower}

A high discharge axial flow fan or a centrifugal blower can be used for sucking and forcing air into the duct. The blower must be able to maintain sufficient pressure so that the air will be able to flow freely through the venturi arrangement.

\subsection{Positive Displacement Pump}

A positive displacement pump used in reverse osmosis water filters is used to raise the pressure of water above 2 bars. This pressure is required for the water to pass through the atomizing nozzle.

\subsection{Atomizing Nozzle}

An atomizing nozzle is used to atomize the water entering the air stream [1]. The nozzle works at a pressure of about 2 bars. Water enters the nozzle through a pressure tube from a positive displacement pump. The nozzle atomizes the water and sprays it as a fine mist inside the duct. Water is sprayed into the air stream before the stream enters the venturi arrangement.

\subsection{Copper Tubes}

Coiled copper tubes are placed after the venturi arrangement. The tubes circulate water through the room to be cooled. There is a separate coil inside the room. This coil is placed in a location such that air that enters the conventional air conditioner cooling coil is at a lower temperature than room temperature. The tubes circulating cold water from the ecofriendly air conditioning system can be coupled with a conventional air conditioner such that the air coming in contact with the conventional air conditioner's cooling coil is already at a lower temperature than the room temperature.

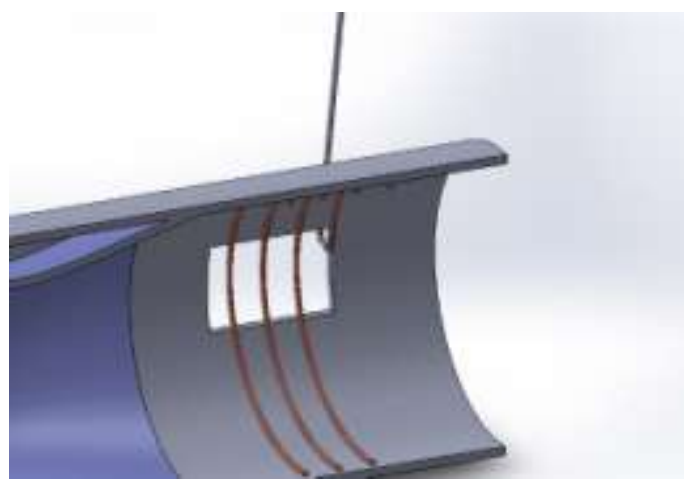

Fig -3: Embedded copper tubes

\subsection{Overall System}

The overall system has a PVC duct into which a small venturi arrangement is built as shown in figure. An atomizing nozzle is inserted before the venturi arrangement. Air at sufficient pressure is forced into the duct. Air enters the duct before the nozzle and venturi arrangement. A coiled copper tube is placed after the venturi arrangement. Warm water from the room enters the copper coils through one end and leaves the system as cold water through the other end. 


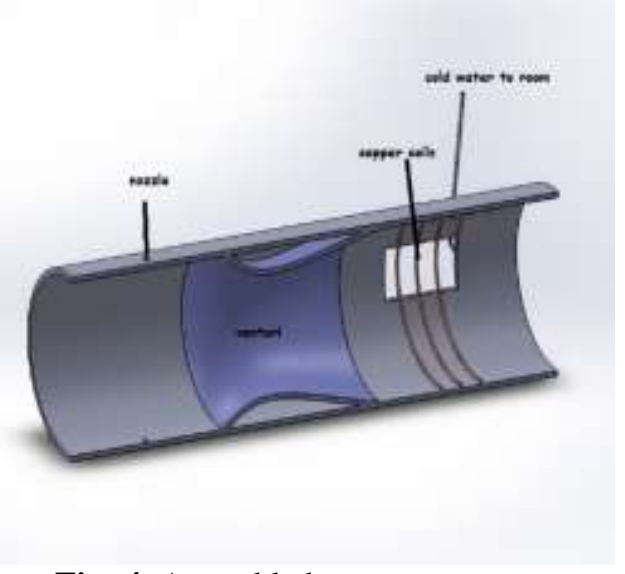

Fig -4: Assembled setup

\section{WORKING}

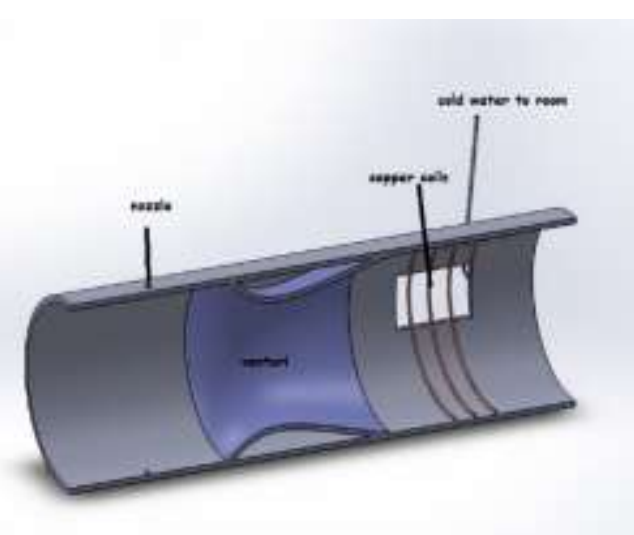

Fig -5: Sectional view

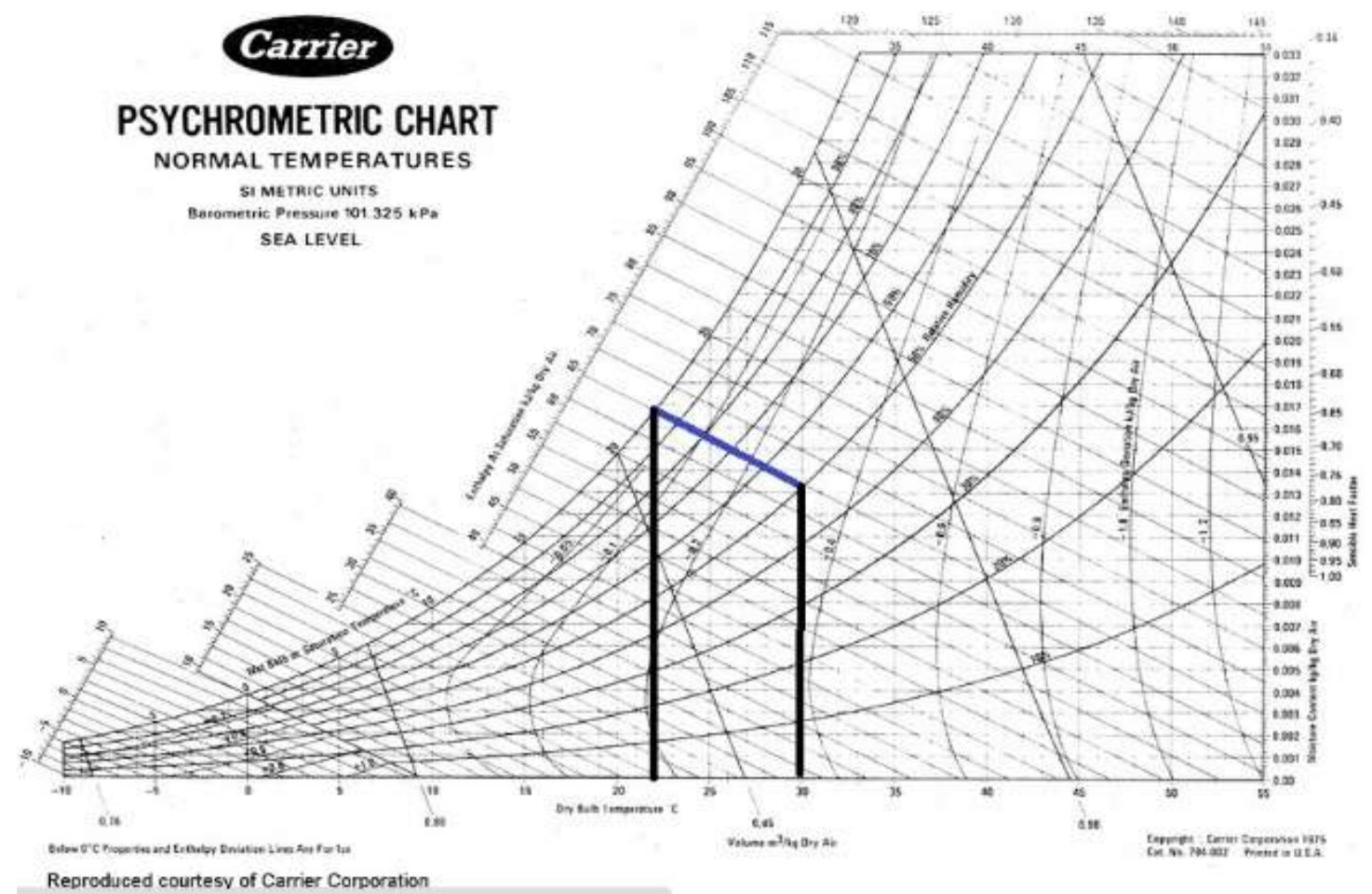

Chart -1: Psychrometric process

Fig -6: Working setup
Air from the blower/axial flow fan enters one end of the duct. Water gets sprayed into the duct. As the water evaporates and saturates the air it will lower the temperature of the air. This can be showed using the psychrometric process shown in figure below:

Assuming the room to be at 30 degree Celsius with $50 \%$ relative humidity

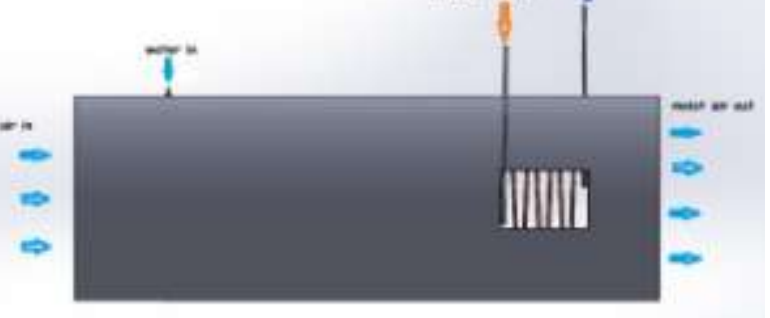


The room is initially at 30 degree Celsius with $50 \%$ relative humidity. When air at this condition is fully saturated the temperature drops to about 22 degree Celsius. This process is shown by the blue line in the figure. Cold air at 22 degree Celsius and $100 \%$ relative humidity now passes through the venturi arrangement. At the throat section of the venture the air pressure drops [4]. This drop in pressure causes more water to evaporate in the air stream this will further reduce the air temperature.

Now cold air at temperatures below 22 degree Celsius comes in contact with the copper tubes carrying water. The water in the copper tubes is circulated through the renewable air conditioning system and through the conventional air conditioning system. Warm water enters the copper tubes at one end and leaves as cold water through the other end. This cooling happens due to the cold air from the venture arrangement coming in contact with the copper tubes. Then the moist air leaves the system.

Cold water from the copper tubes is taken to the room and passed through another copper coil places before the air intake of a conventional air conditioning system. Thus the air entering the conventional air conditioning system is already at a precooled condition. This will greatly decrease the air conditioner's power requirements.

\subsection{Energy Requirements}

If a room at 30 degree Celsius and $50 \%$ relative humidity is considered

For normal air conditioning:

Amount of energy to be removed from room

$=85 \mathrm{KJ} / \mathrm{kg}$ of dry air $-40 \mathrm{KJ} / \mathrm{kg}$ of dry air

$=45 \mathrm{KJ} / \mathrm{kg}$ of dry air

For normal air conditioning coupled with eco-friendly air conditioner:

Amount of energy to be removed from room

$=55 \mathrm{KJ} / \mathrm{kg}$ of dry air $-40 \mathrm{KJ} / \mathrm{kg}$ of dry air

$=15 \mathrm{KJ} / \mathrm{kg}$ of dry air

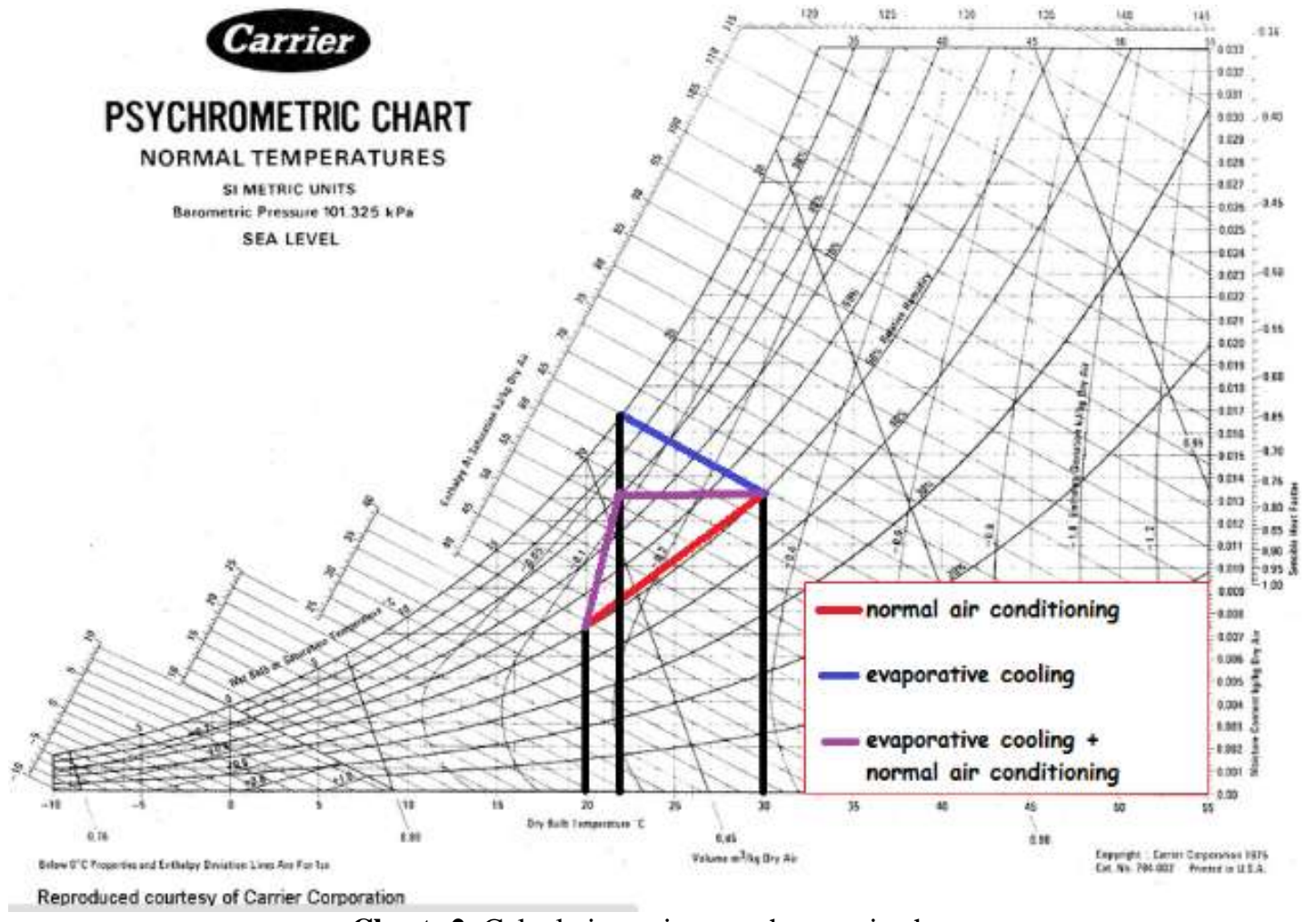

Chart -2: Calculation using psychrometric chart

From the above calculations we can come to an inference that for the same room to cool the room from 30 degree Celsius and $50 \%$ relative humidity to 20 degree Celsius and $50 \%$ relative humidity, by coupling a eco-friendly air conditioning system, the amount of heat to be removed from the room decreases by $66.67 \%$. This means that to achieve the same cooling effect a conventional air conditioning system of reduced tonnage can be used.
By employing this system, the power consumption for room cooling can be greatly reduced. This is because a lower power compressor is enough to run the system now and the power consumed by the components used in the eco-friendly system is less than 100 watts. Therefore by employing such systems in house cooling the electricity usage can be greatly reduced. This also reduces carbon emissions and protects our environment. 


\section{CONCLUSION}

The design and fabrication of an eco-friendly air conditioning system is discussed in this paper. This system can be fabricated using simple components available. It is very cheap and it can be attached to a conventional air conditioning system. Attaching this system to a conventional air conditioning system will greatly increase the efficiency of operation of the conventional system and will also reduce the power consumption of these systems. These systems have an added advantage in hot and dry climatic conditions. Hot and dry conditions will accelerate evaporative cooling and will improve the cooling effect. By using this system we can reduce the power consumption of conventional air conditioning systems. By reducing the power consumption, the carbon emissions can also be reduced. An air conditioner with a reduced capacity can be used when it is coupled with the eco-friendly air conditioning system. Thus the usage of this system can be recommended for cooling living spaces and commercial spaces. They can also be used in vehicles such that the cooling effect does not absorb much of the engines power and hence the efficiency of the engine is also increased.

\section{REFERENCES}

[1] Karl Johnson, "Fundamentals of Thermodynamics", University of Pittsburgh, 2009.

[2] R.K. Rajput, "Engineering Thermodynamics", Firewell Media, 2010.

[3] P.K. Nag, "Engineering Thermodynamics", Tata McGraw- Hill Education, 2013.

[4] Yunus Cengel and Michael Boles, "Thermodynamics: An Engineering approach”, McGraw- Hill Education. 